is an essential characteristic of people with a high standard of physical fitness with regard to endurance ${ }^{6}$.

Department of Physiology,

Kungliga Gymnastiska Centralinstitutet, Stockholm.

June 1 .

${ }^{1}$ Robinson, S., Edwards, H. T., and Dill, D. B., Science, 85, 409 (1937). 2 Robinson, S., Arbeitsphysiologie, 10, 251 (1938).

${ }^{3}$ Astrand, P.-O., "Experimental Studies of Physical Working Capacity in Relation to Sex and Age" (Munksgaard, Copenhagen, 1952). 4 Astrand, P.-0., Arbeitsphysiologie, 15, 251 (1953).

- Astrand, P.-O., and Ryhming, Irma, J. App. Physiol., 7, 218 (1954). - Herbst, R., Dtsch. Arch. klin, Med., 162, 33 (1928). Bock, A. V.. Vancaulaert, C., Dill, D. B., Fölling, A., and Hurxthal, L. M., Astrand, P.-0., FIEP-Bulletin, 24, 52 (1954).

\section{Corona Wind Loud-speaker}

Experiments made with a pair of electrodes, one sharp and one blunt, show that a corona wind moves away from the sharp point independent of its polarity. If the needle is positive, the wind is stronger than when it is negative.

The wind may be controlled by placing a blunt ring or 'grid' around the sharp electrode: if this ring is maintained at the same order of voltage as the sharp electrode, then there is a position of the latter at which comparatively small changes of the grid potential produce a maximum effect on the intensity of the corona wind and hence on the corona

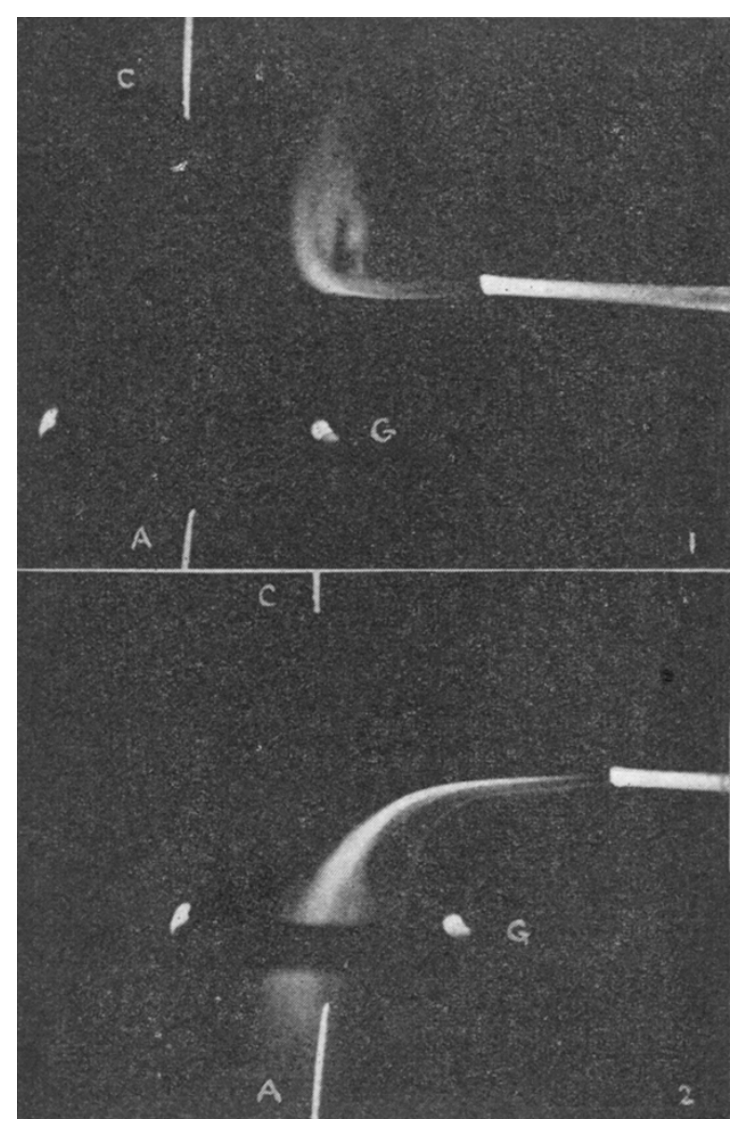

(1) Strong positive wind from needle $A ; \nabla_{A} \sigma=17.5 \mathrm{kV}$. and $V_{G O}=8 \cdot 5 \mathrm{kV}$. (2) Strong negative wind from needle $C$;
$\nabla_{A O}=18 \cdot 6 \mathrm{kV}$. and $\nabla G O=15.2 \mathrm{kV}$. current. This may be explained on the assumption that lines of force which otherwise would terminate on the tip of the needle are deflected to the grid ring to an extent dependent on the relative potentials of the electrodes. Thus although the grid takes no current itself, variations of its potential can control the corona current over a wide range. An extensive experimental study of this phenomenon has been made and will be published elsewhere.

With two sharp electrodes $A, C$ (Fig. 1) opposed to each other, the strong positive wind greatly exceeds the negative. The interaction of the two winds causes an injected smoke stream from the region of the positive needle to avoid the negative one in an umbrella-shaped form (not shown) near the negative needle. If now the ring electrode $G$ is placed around the positive needle $A$, the positive wind may be completely cut off (Fig. 2), in which case the negative wind predominates. With a positive wind adjusted to equality with the negative there is no net wind.

An alternating potential applied to the grid causes the air to pulsate and hence to become a source of sound.

With a stacked array of triodes large enough to give a reasonable acoustic area, the device becomes a loud-speaker with certain distinctive features. (a) It has no moving parts. (b) It requires no electrical power at acoustic frequencies to drive it, since the 'grid' ring takes no current. The power comes from a d.c. source across the needles. (c) It has a good bass response, without any bass resonance even down to zero frequency: the steady wind is the zerofrequency response. (d) It has a good middle- and high-frequency response smooth to $15 \mathrm{kc} . / \mathrm{s}$. (e) In its present form it has a severe limitation in its linearity; but a push-pull form is under construction. (f) Its power output under an approximately linear adjustment is about $0.1 \mathrm{~mW}$. per sq. cm. of matrix area, a figure comparable with types of electrostatic loud-speaker using diaphragms. The d.c. power input is about $50 \mathrm{~mW}$. per sq. cm. at $12 \mathrm{kV}$. (g) The polar distribution of sound may be varied at will by varying the shape of the matrix. It does not need to be planar.

Electrical Engineering Department,

D. M. TOMBs

Imperial College, London, S.W.7. July 6.

\section{Magnetic Moment of an Excited State of the Fluorine-19 Nucleus}

ThHRE is a well-known technique ${ }^{1}$ for the measurement of magnetic moments of short-lived nuclear states. A strong external magnetic field applied to a radioactive source which emits $\gamma$-rays in a cascade process will influence the nuclear spin of the intermediate state, and thus change the $\gamma-\gamma$ angular correlation. The strength of the interaction is a measure of the nuclear moment, and the theory of the process has been worked out ${ }^{2,3}$.

The same principle has been applied to a study of the angular distribution of $\gamma$-rays resulting from the nuclear reaction :

$$
p+{ }^{10} \mathrm{~F} \rightarrow p^{\prime}+{ }^{19} \mathrm{~F}^{* *} \rightarrow p^{\prime}+\gamma+{ }^{19} \mathrm{~F}
$$

where the intermediate nucleus $\left({ }^{19} \mathrm{~F}^{* *}\right)$ is known ${ }^{4}$ to have a lifetime of $(1 \cdot 0 \pm 0.2) \times 10^{-7}$ sec., spin $5 / 2$ and excitation $197 \mathrm{keV}$. A uniform vertical magnetic field was applied normal to the beam of the Canberra H.T. set, and protons of $873 \mathrm{keV}$. were allowed to 